\title{
Sleep and Hypnotics: Further Experiments
}

\author{
J. I. EVANS, ${ }^{*}$ M.B., CH.B., D.P.M.; O. OGUNREMI, $\dagger$ M.B., B.S.
}

Cummary: Two experiments compatible with that used to investigate the effect of clinical doses $(200 \mathrm{mg}$.) of amylobarbitone were set up to investigate the effects of clinical doses of chloral hydrate $(800 \mathrm{mg}$.), dichloral phenazone (1,300 mg.), and Mandrax (methaqualone $250 \mathrm{mg}$. and diphenhydramine $25 \mathrm{mg}$.) over a period of one to two weeks.

Four healthy male subjects were used in each experiment and received placebo or drug throughout a period of six to eight weeks when control records, drug records, and drug withdrawal records were obtained.

Chloral hydrate was found to depress rapid eye movement (R.E.M.) sleep appreciably though less consistently than amylobarbitone. No withdrawal R.E.M. sleep rebound was found.

Neither dichloralphenazone nor Mandrax was found consistently to depress R.E.M. sleep, though occasional nights when R.E.M. sleep was low occurred more often with Mandrax.

In the light of other experiments it is postulated that there exists a "threshold" in the dose of a hypnotic, and that when this is exceeded the drug will produce R.E.M. reduction. Thus it may be possible to prescribe a drug which is clinically useful while avoiding withdrawal effects.

\section{Introduction}

The great expansion during the past 15 years of information on the measurement of sleep has prompted further research into the effects of hypnotic drugs (Oswald and Priest, 1965; Evans et al., 1968; Kales et al., 1968, 1969).

Sleep is composed of two regularly alternating physiological states: (1) orthodox sleep, characterized by regularity of physiological indices and relative absence of mental activity, and (2) paradoxical or rapid eye movement (R.E.M.) sleep, in which there is great irregularity of many physiological measures and in which the bulk of dreaming occurs (Dement and Kleitman, 1957; Jouvet, 1967). Orthodox sleep is further divided into four stages, and during much of adult life R.E.M. sleep is usually found to occupy about $24 \%$ of total sleep.

Sodium amylobarbitone has been the drug most used to examine the effects of a barbiturate on sleep (Oswald and Priest, 1965; Evans et al., 1968). In clinical doses it produced an immediate alteration in the relationship of orthodox sleep to R.E.M. sleep. Orthodox sleep was enhanced and there was considerable depression of R.E.M. sleep. During continued administration the amount of R.E.M. sleep approximated towards baseline values-a "tolerance" phenomenon. Stopping the drug produced a fluctuant excess of R.E.M. sleep which subsided over several weeks-a "rebound" phenomenon-and in the larger dose experiments this R.E.M. sleep excess was associated with nightmares (Oswald and \footnotetext{
* Lecturer and Nuffield Scholar, Department of Psychiatry, Edinburgh
University, Edinburgh EH10 5HF.

University, Edinburgh EH10 $5 \mathrm{HF}$.
+ Registrar, Royal Edinburgh Hospital.
}

Priest, 1965). This persistent state may explain the difficulties experienced in withdrawing the drug in some patients.

Though many patients may continue with the drug for long periods without attendant difficulties (Johnson and Clift, 1968), it would seem desirable that hypnotics should be free from withdrawal effects if this is possible.

The effects of various non-barbiturate hypnotics on sleep have been studied, but results are conflicting. Oswald and Priest (1965) found that $15 \mathrm{mg}$. of nitrazepam did not differ from amylobarbitone in its effects on R.E.M. sleep, though Tissot (1965) reported that nitrazepam enhanced R.E.M. sleep. Kales et al. (1968) showed that glutethimide $500 \mathrm{mg}$. and methyprylone $300 \mathrm{mg}$. also depressed R.E.M. sleep, and their withdrawal was accompanied by a R.E.M. sleep rebound. Kales et al. (1969) reported that while $300 \mathrm{mg}$. of methaqualone depressed R.E.M. sleep $150 \mathrm{mg}$. of the drug had no appreciable effects. Davison (1969) stated that Mandrax, a combination drug containing methaqualone $250 \mathrm{mg}$. and diphenhydramine $25 \mathrm{mg}$., also had no significant effect on R.E.M. sleep. Kales et al. (1969) reported that chloral hydrate in doses of $500 \mathrm{mg}$. and $1 \mathrm{~g}$. did not appreciably affect R.E.M. sleep. Lehmann and Ban (1968) found that chloral hydrate (650 mg.) disturbed R.E.M. sleep insignificantly in comparison with quinalbarbitone (secobarbital) (100 mg.) or nitrazepam (10 mg.).

These observations raise some questions in relation to a current theory of drug withdrawal delirium (Gross et al., 1966; Greenberg and Pearlman, 1967). Drugs which on withdrawal can give rise to delirium-that is, alcohol, barbiturates and other hypnotics, meprobamate-have all been shown to produce R.E.M. depression and to be associated with an R.E.M. rebound in withdrawal (Gresham et al., 1963; Evans and Lewis, 1968; Oswald et al., 1969). It is an old observation (de Clérambault, 1910) that when chloral hydrate was withdrawn from addicts delirium resulted. This strongly suggests that chloral hydrate disturbs R.E.M. sleep.

In view of these conflicts two separate experiments were arranged to examine the effects of clinical doses of chloral hydrate, dichloralphenazone, and Mandrax (methaqualone $250 \mathrm{mg}$. and diphenhydramine $25 \mathrm{mg}$.).

\section{Method}

The experimental design follows closely that used to examine the effects of sodium amylobarbitone in clinical doses (Evans et al., 1968).

Four healthy men aged 21 to 26 were used in each experiment. Throughout the experimental period, six to eight weeks, they were instructed to refrain from alcohol and any medication and to keep regular hours when not recorded. The first recorded night in the laboratory was regarded as a "habituation" night (Mendels and Hawkins, 1967) and discarded. A series of six nights taken at intervals over a fortnight were used as baseline data. Five to eight intermittent night recordings were taken when the subjects were on hypnotics, and a similar number of recordings were obtained in the withdrawal period (for details see Table I and II).

On a recording night subjects reported to the laboratory at 22.30 hours. Silver disc electrodes were attached round the 
eyes, to the scalp in the midline, and under the chin as described elsewhere (Evans et al., 1968). Bipolar montages were set up to record eye movements, encephalogram, and muscle tone on an encephalograph which ran continuously throughout the night. The record was analysed according to internationally agreed criteria (Rechtschaften and Kales, 1968).

Experiment 1.-The subjects received cachets of either placebo or active drug every night throughout the experiment, whether they were recorded or not. Sodium lactose was used as a placebo, and $0.8 \mathrm{~g}$. of chloral hydrate was chosen as a convenient dose of drug. Unfortunately Subject $C$ developed a peridontal abscess during the late control period, and he and Subject $\mathrm{D}$ were therefore left on placebo for a further week as Subject $C$ required other medication. Subjects $A$ and $B$ received the drug for 13 consecutive nights while Subjects $C$ and $D$ took the drug for six nights. The withdrawal period on placebo was monitored for 13 nights. Cachets were taken at 23.00 hours.

Experiment 2.-After a series of habituation and baseline nights on placebo, four different subjects received either dichloralphenazone $(1.3 \mathrm{~g})$ or Mandrax by double-blind design. The drug was continued for 14 nights and a further series of records were obtained during withdrawal while the subjects were again on placebo. All cachets were taken at 23.00 hours.

\section{Results}

Experiment 1.-The data are presented in Table I. Chloral hydrate was found to disturb the balance of orthodox to R.E.M. sleep. R.E.M. sleep was depressed in all four subjects during the first two or three nights on the drug, and this depression was found to be significant at the 0.5 level $(t=13.6222)$ with the statistical procedure outlined previously (Lewis and Evans, 1969). This R.E.M. depressing effect was found usually in the first two to three hours of sleep, leading to an increased delay to the first R.E.M. period and absence of R.E.M. activity in the first two hours of sleep. Occasional later nights show R.E.M. depression but there is overall a return to baseline values. This drop in R.E.M. sleep is accompanied by an increase in stages I and II of orthodox sleep and there is no evidence of changes on stages III and IV of orthodox sleep. Taking all four subjects together there is a tendency for the number of arousals and time awake to decrease on the drug, but there was no discernible alteration in the delay to sleep (d).

Experiment 2.-The data are outlined in Table II. Neither drug in clinical dose had pronounced or consistent effects on R.E.M. sleep. Subjects A and B had isolated nights when R.E.M. sleep was reduced, but there were no overall significant changes in the dimensions. Because of an increase in fast activity in the electroencephalograms of Subjects A and B in association with these low R.E.M. sleep nights, it was obvious that the drug received by Subjects A and B was different from that received by Subjects $C$ and $D$. Correspondingly Subject B had a significantly raised R.E.M. sleep percentage on the first withdrawal night and Subject $C$ had a raised R.E.M. sleep time on the second withdrawal night, though no consistent withdrawal state was found.

\section{Discussion}

In clinical doses these hypnotics disturb the balance of orthodox and R.E.M. sleep less than a clinical dose of

TABLE I-Experiment 1. Effect of $0.8 \mathrm{~g}$. Chloral Hydrate on Sleep

\begin{tabular}{|c|c|c|c|c|c|c|c|c|c|c|}
\hline \multicolumn{4}{|c|}{ Record } & \multirow[t]{2}{*}{ Subject } & \multirow[t]{3}{*}{$\begin{array}{c}\text { Total Sleep Time } \\
\text { (min.) }\end{array}$} & \multirow[t]{2}{*}{$\mathrm{d}(\min )}$. & \multirow[t]{2}{*}{ D $\underset{(\text { min.) }}{\text { (R.E.M.) }}$} & \multirow[t]{2}{*}{$\begin{array}{c}\text { Stages } \\
\text { III + IV (\%) }\end{array}$} & \multirow[t]{2}{*}{$\begin{array}{r}\text { R.E.M. } \\
\text { Sleep (\%) }\end{array}$} & \multirow[t]{2}{*}{$\begin{array}{l}\text { Shifts to Stage I } \\
\text { or Awake/Hour }\end{array}$} \\
\hline \multicolumn{4}{|c|}{ Subjects $A$ and $B$} & & & & & & & \\
\hline $\begin{array}{l}\text { Contro } \\
\text { Contro } \\
\text { Contro } \\
\text { Contro } \\
\text { Contro } \\
\text { Contro } \\
\end{array}$ & & $\begin{array}{l}. \\
\because \\
\cdots \\
\cdots \\
.\end{array}$ & $\begin{array}{l}. \\
\therefore \\
\cdots \\
\therefore \\
\therefore\end{array}$ & $\begin{array}{l}\mathbf{A} / \mathbf{B} \\
\mathbf{A} / \mathbf{B} \\
\mathbf{A} / \mathbf{B} \\
\mathbf{A} / \mathbf{B} \\
\mathbf{A} / \mathbf{B} \\
\mathbf{A} / \mathbf{B} \\
\end{array}$ & & $\begin{array}{r}9 / 16 \\
13 / 28 \\
5 / 30 \\
2 / 3 \\
4 / 14 \\
60 / 2 \\
\end{array}$ & $\begin{array}{r}157 / 154 \\
95 / 86 \\
75 / 72 \\
143 / 151 \\
74 / 71 \\
56 / 55\end{array}$ & $\begin{array}{l}20 \cdot 0 / 21 \cdot 6 \\
21 \cdot 4 / 20 \cdot 8 \\
29 \cdot 8 / 22 \cdot 8 \\
27 \cdot 4 / 23 \cdot 7 \\
26 \cdot 8 / 31 \cdot 5 \\
15 \cdot 6 / 21 \cdot 9\end{array}$ & $\begin{array}{l}21 \cdot 7 / 21 \cdot 3 \\
22 \cdot 5 / 24 \cdot 0 \\
22 \cdot 0 / 27 \cdot 1 \\
21 \cdot 0 / 24 \cdot 8 \\
20 \cdot 6 / 24 \cdot 4 \\
24 \cdot 6 / 26 \cdot 4\end{array}$ & $\begin{array}{l}4 \cdot 00 / 4 \cdot 50 \\
3 \cdot 83 / 3 \cdot 66 \\
5 \cdot 33 / 4 \cdot 66 \\
4 \cdot 00 / 3 \cdot 33 \\
4 \cdot 16 / 4 \cdot 16 \\
4 \cdot 33 / 3 \cdot 66\end{array}$ \\
\hline $\begin{array}{l}\text { Drug } \\
\text { Drug } \\
\text { Drug } \\
\text { Drug } \\
\text { Drug } \\
\text { Drug } \\
\text { Drug } \\
\text { Drug }\end{array}$ & $\begin{array}{r}\text { I } \\
\text { II } \\
\text { III } \\
\text { V } \\
\text { VII } \\
\text { IX } \\
\text { XI } \\
\text { XIII }\end{array}$ & $\begin{array}{l}. \\
\because \\
\because \\
\because \\
\because \\
\cdots\end{array}$ & $\begin{array}{l}. . \\
. . \\
\because \\
. \\
\therefore \\
. .\end{array}$ & $\begin{array}{l}\mathbf{A} / \mathbf{B} \\
\mathbf{A} / \mathbf{B} \\
\mathbf{A} / \mathbf{B} \\
\mathbf{A} / \mathbf{B} \\
\mathbf{A} / \mathbf{B} \\
\mathbf{A} / \mathbf{B} \\
\mathbf{A} / \mathbf{B} \\
\mathbf{A} / \mathbf{B}\end{array}$ & $\begin{array}{l}458 / 474 \\
467 / 472 \\
458 / 472 \\
469 / 469 \\
474 / 482 \\
438 / 438 \\
470 / 462 \\
469 /-\end{array}$ & 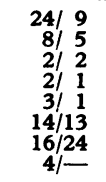 & $\begin{array}{c}147 / 143 \\
170 / 142 \\
77 / 134 \\
56 / 131 \\
60 / 125 \\
64 / 163 \\
59 / / 53 \\
71 /-\end{array}$ & $\begin{array}{l}21 \cdot 9 / 23 \cdot 8 \\
20 \cdot 3 / 24 \cdot 8 \\
24 \cdot 4 / 17 \cdot 1 \\
20 \cdot 4 / 34 \cdot 4 \\
26 \cdot 5 / 28 \cdot 8 \\
15 \cdot 5 / 19 \cdot 5 \\
27.4 / 14 \cdot 0 \\
39 \cdot 2 /-\end{array}$ & $\begin{array}{l}13 \cdot 6 / 17 \cdot 5 \\
14.9 / 15 \cdot 8 \\
15 \cdot 5 / 21 \cdot 6 \\
23 \cdot 6 / 14 \cdot 3 \\
21 \cdot 4 / 19 \cdot 3 \\
14 \cdot 4 / 17 \cdot 5 \\
18 \cdot 3 / 19 \cdot 3 \\
17 \cdot 4 /-\end{array}$ & $\begin{array}{l}5 \cdot 83 / 2 \cdot 86 \\
5 \cdot 10 / 3 \cdot 50 \\
4 \cdot 33 / 2 \cdot 50 \\
3 \cdot 50 / 3 \cdot 50 \\
4 \cdot 63 / 3 \cdot 33 \\
6 \cdot 33 / 3.50 \\
3 \cdot 83 / 3 \cdot 33 \\
3 \cdot 33 /-\end{array}$ \\
\hline Off & $\begin{array}{r}\text { I } \\
\text { II } \\
\text { III } \\
\text { V } \\
\text { VII } \\
\text { IX } \\
\text { XI } \\
\text { XIII } \\
\end{array}$ & $\begin{array}{l}. \\
\because \\
\because \\
\cdots \\
\cdots \\
\cdots\end{array}$ & 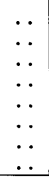 & $\begin{array}{l}\mathbf{A} / \mathbf{B} \\
\mathbf{A} / \mathbf{B} \\
\mathbf{A} / \mathbf{B} \\
\mathbf{A} / \mathbf{B} \\
\mathbf{A} / \mathbf{B} \\
\mathbf{A} / \mathbf{B} \\
\mathbf{A} / \mathbf{B} \\
\mathbf{A} / \mathbf{B} \\
\end{array}$ & $\begin{array}{l}452 / 472 \\
459 / 460 \\
456 / 436 \\
469 /- \\
452 /- \\
460 / 492 \\
446 / 447 \\
470 / 467 \\
\end{array}$ & $\begin{array}{l}13 / 11 \\
30 / 19 \\
10 / 30 \\
20 /- \\
8 /= \\
20 / 10 \\
8 / 7 \\
12 / 9 \\
\end{array}$ & $\begin{array}{c}138 / 60 \\
62 / 57 \\
81 / 64 \\
92 /- \\
63 / \overline{6} \\
69 / 66 \\
63 / 49 \\
58 / 66 \\
\end{array}$ & $\begin{array}{l}18 \cdot 1 / 18 \cdot 9 \\
28 \cdot 9 / 16 \cdot 7 \\
23 \cdot 8 / 22 \cdot 6 \\
22 \cdot 9 /= \\
22 \cdot 8 / \\
22 \cdot 7 / 22 \cdot 2 \\
27 \cdot 3 / 18 \cdot 8 \\
31 \cdot 1 / 14 \cdot 0 \\
\end{array}$ & $\begin{array}{l}14 \cdot 2 / 26 \cdot 0 \\
19 \cdot 0 / 22 \cdot 4 \\
23 \cdot 1 / 19 \cdot 1 \\
25 \cdot 7 /= \\
18 \cdot 4 ! \\
20.8 / 21 \cdot 4 \\
19 \cdot 7 / 25 \cdot 4 \\
20 \cdot 4 / 23 \cdot 6 \\
\end{array}$ & $\begin{array}{l}6 \cdot 16 / 4 \cdot 14 \\
5 \cdot 66 / 3 \cdot 33 \\
4 \cdot 00 / 3.50 \\
5 \cdot 00 / / \\
4 \cdot 50 /- \\
3.83 / 4 \cdot 83 \\
4 \cdot 13 / 3.50 \\
3.33 / 4 \cdot 00 \\
\end{array}$ \\
\hline \multicolumn{11}{|c|}{ Subjects $C$ and $D$} \\
\hline $\begin{array}{l}\text { Contro } \\
\text { Contro } \\
\text { Contro } \\
\text { Contro } \\
\text { Control } \\
\text { Contro } \\
\end{array}$ & & $\begin{array}{l}. \\
\cdots \\
\cdots \\
\cdots \\
.\end{array}$ & $\begin{array}{l}. \\
\therefore \\
\therefore \\
\cdots \\
\cdots\end{array}$ & $\begin{array}{l}\text { C/D } \\
\mathbf{C} / \mathbf{D} \\
\mathbf{C} / \mathbf{D} \\
\mathbf{C} / \mathbf{D} \\
\mathbf{C} / \mathbf{D} \\
\mathbf{C} / \mathbf{D}\end{array}$ & $\begin{array}{l}468 / 400 \\
370 / 402 \\
432 / 423 \\
457 / 419 \\
447 / 426 \\
448 / 469\end{array}$ & $\begin{array}{l}7 / 6 \\
83 / 11 \\
65 / 9 \\
14 / 9 \\
33 / 4 \\
12 / 14\end{array}$ & $\begin{array}{c}128 / 64 \\
58 / 77 \\
166 / 58 \\
80 / 160 \\
64 / 80 \\
73 / 67\end{array}$ & $\begin{array}{l}38 \cdot 1 / 44 \cdot 4 \\
35 \cdot 7 / 31 \cdot 9 \\
27 \cdot 1 / 31 \cdot 6 \\
33 \cdot 4 / 35 \cdot 7 \\
28 \cdot 4 / 28 \cdot 2 \\
28 \cdot 0 / 33 \cdot 9\end{array}$ & $\begin{array}{l}24 \cdot 2 / 19 \cdot 8 \\
21 \cdot 0 / 20 \cdot 5 \\
20 \cdot 9 / 22 \cdot 8 \\
24 \cdot 9 / 16 \cdot 0 \\
24 \cdot 2 / 17 \cdot 3 \\
25 \cdot 1 / 19 \cdot 6\end{array}$ & $\begin{array}{l}2 \cdot 00 / 1 \cdot 66 \\
1.50 / 3.50 \\
1.16 / 2.50 \\
1.66 / 1.83 \\
1.33 / 2 \cdot 16 \\
2 \cdot 50 / 2.00\end{array}$ \\
\hline $\begin{array}{l}\text { Drug } \\
\text { Drug } \\
\text { Drug } \\
\text { Drug } \\
\text { Drug } \\
\end{array}$ & $\begin{array}{r}\text { I } \\
\text { II } \\
\text { III } \\
\mathbf{V} \\
\text { VII } \\
\end{array}$ & $\begin{array}{l}. \\
\cdots \\
\cdots \\
\cdots \\
\end{array}$ & $\begin{array}{l}\cdots \\
\cdots \\
\cdots \\
\cdots\end{array}$ & $\begin{array}{l}\text { C/D } \\
\text { C/D } \\
\text { C/D } \\
\text { C/D } \\
\text { C/D }\end{array}$ & $\begin{array}{l}434 / 401 \\
436 / 423 \\
466 / 444 \\
432 / 436 \\
462 / 477\end{array}$ & $\begin{array}{l}16 / 21 \\
36 / 13 \\
19 / 16 \\
41 / 4 \\
20 / 4\end{array}$ & $\begin{array}{r}73 / 143 \\
63 / 70 \\
112 / 55 \\
62 / 180 \\
62 / 175\end{array}$ & $\begin{array}{l}25 \cdot 0 / 33 \cdot 5 \\
26 \cdot 2 / 31 \cdot 6 \\
21 \cdot 6 / 20 \cdot 8 \\
31 \cdot 0 / 39 \cdot 5 \\
45 \cdot 1 / 29 \cdot 1\end{array}$ & $\begin{array}{l}19 \cdot 3 / 15 \cdot 0 \\
16 \cdot 7 / 8 \cdot 7 \\
19 \cdot 7 / 14 \cdot 3 \\
27 \cdot 0 / 10 \cdot 8 \\
20 \cdot 4 / 18 \cdot 1\end{array}$ & $\begin{array}{l}0 \cdot 83 / 3 \cdot 16 \\
1 \cdot 50 / 2 \cdot 33 \\
2 \cdot 33 / 2 \cdot 66 \\
1 \cdot 00 / 1 \cdot 33 \\
1 \cdot 00 / 2 \cdot 33\end{array}$ \\
\hline Off & $\begin{array}{r}\text { I } \\
\text { II } \\
\text { III } \\
\text { V } \\
\text { VII } \\
\text { IX } \\
\text { XI } \\
\text { XIII }\end{array}$ & $\begin{array}{l}\ldots \\
\cdots \\
\cdots \\
\cdots \\
\cdots \\
\cdots\end{array}$ & $\begin{array}{l}\ldots \\
\ldots \\
\ldots \\
\ldots \\
\ldots \\
\ldots\end{array}$ & $\begin{array}{l}\text { C/D } \\
\text { C/D } \\
\text { C/D } \\
\text { C/D } \\
\text { C/D } \\
\text { C/D } \\
\text { C/D } \\
\text { C/D }\end{array}$ & $\begin{array}{l}459 / 441 \\
447 / \\
440 / 426 \\
476 / 450 \\
450 / 444 \\
-/ 429 \\
441 / 424 \\
446 / 419\end{array}$ & $\begin{array}{l}24 / 11 \\
39 / 1 \\
22 / 6 \\
13 / 2 \\
2 / 11 \\
3 / 6 \\
15 / 14 \\
24 / 12\end{array}$ & $\begin{array}{r}67 / \\
62 / / 56 \\
171 / 58 \\
64 / 64 \\
44 / 95 \\
-/ 68 \\
60 / / 96 \\
69 / 205\end{array}$ & $\begin{array}{l}30 \cdot 7 / 30 \cdot 1 \\
28 \cdot 0 / \\
39 \cdot 1 / 30 \cdot 5 \\
38 \cdot 2 / 34 \cdot 3 \\
35 \cdot 7 / 30 \cdot 5 \\
-132 \cdot 2 \\
46 \cdot 1 / 37 \cdot 0 \\
44 \cdot 1 / 32 \cdot 6\end{array}$ & $\begin{array}{r}23 \cdot 4 / 21 \cdot 9 \\
22 \cdot 8 / \\
18 \cdot 3 / 17 \cdot 7 \\
29 \cdot 2 / 18 \cdot 1 \\
29 \cdot 6 / 15 \cdot 1 \\
-/ 20 \cdot 4 \\
24 \cdot 5 / 21 \cdot 4 \\
26 \cdot 8 / 12 \cdot 9\end{array}$ & $\begin{array}{l}1.50 / 2.50 \\
1.83 / 2 \\
2.63 / 2.66 \\
1.62 / 3.33 \\
3.00 / 2.66 \\
-/ 1.50 \\
1.16 / 3.33 \\
1.13 / 2.50\end{array}$ \\
\hline
\end{tabular}


TABLE II-Experiment 2

\begin{tabular}{|c|c|c|c|c|c|c|c|c|}
\hline \multicolumn{2}{|l|}{ Record } & Subiect & $\underset{\substack{\text { (min) } \\
\text { Total }}}{\text { Slep Time }}$ & $\mathrm{d}(\min )$. & $\mathbf{D}$ (min.) & $\mid$ Stages III +IV (\%) & R.E.M. Sleep (\%) & $\begin{array}{l}\text { To Stage I or } \\
\text { Awake/Hour }\end{array}$ \\
\hline \multicolumn{9}{|c|}{ Subjects $A$ and $B$} \\
\hline $\begin{array}{ll}\text { Control } & \\
\text { Control } & \because \\
\text { Control } & \because \\
\text { Control } & \\
\text { Control } & \\
\text { Control } & \because\end{array}$ & $\begin{array}{c}: . \\
\because \\
\because: \\
:\end{array}$ & $\begin{array}{l}\mathrm{A} / \mathrm{B} \\
\mathrm{A} / \mathrm{B} \\
\mathrm{A} / \mathrm{B} \\
\mathrm{A} / \mathrm{B} \\
\mathrm{A} / \mathrm{B} \\
\mathrm{A} / \mathrm{B}\end{array}$ & 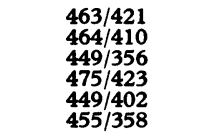 & $\begin{array}{r}9 / 51 \\
1 / 51 \\
11 / 102 \\
3 / 54 \\
7 / 54 \\
4 / 101\end{array}$ & $\begin{array}{l}135 / 45 \\
179 / 66 \\
76 / 37 \\
170 / 54 \\
127 / 42 \\
86 / 30\end{array}$ & 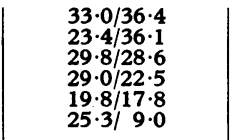 & 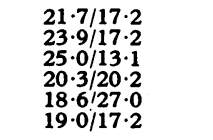 & 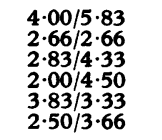 \\
\hline 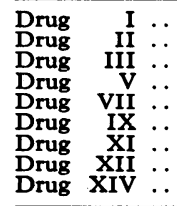 & \begin{tabular}{c|}
$\because$ \\
$\because$ \\
$\because$ \\
$\because$ \\
$\because$ \\
$:$ \\
\end{tabular} & $\begin{array}{l}\mathrm{A} / \mathrm{B} \\
\mathrm{A} / \mathrm{B} \\
\mathrm{A} / \mathrm{B} \\
\mathrm{A} / \mathrm{B} \\
\mathrm{A} / \mathrm{B} \\
\mathrm{A} / \mathrm{B} \\
\mathrm{A} / \mathrm{B} \\
\mathrm{A} / \mathrm{B} \\
\mathrm{A} / \mathrm{B} \\
\mathrm{A} / \mathrm{B}\end{array}$ & 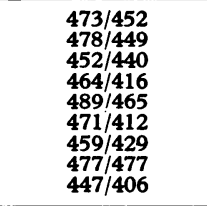 & $\begin{array}{l}3 / 24 \\
5 / 34 \\
4 / 14 \\
3 / 51 \\
7 / 31 \\
22 / 82 \\
5 / 32 \\
5 / 3 \\
14 / 59\end{array}$ & $\begin{array}{c}231 / 62 \\
84 / 91 \\
246 / 113 \\
74 / 47 \\
68 / 66 \\
57 / 58 \\
57 / 58 \\
40 / 15 \\
53 / 153 \\
66 / 52\end{array}$ & 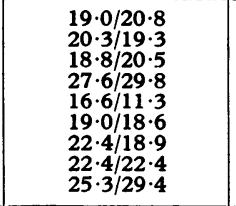 & 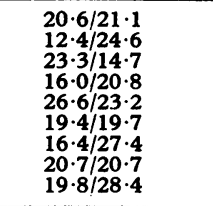 & 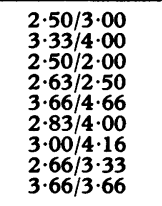 \\
\hline 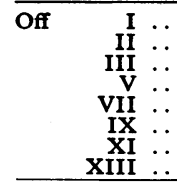 & \begin{tabular}{c|}
$\because$ \\
$\because$ \\
$\because$ \\
$\because$ \\
$\therefore$
\end{tabular} & $\begin{array}{l}\mathrm{A} / \mathrm{B} \\
\mathrm{A} / \mathrm{B} \\
\mathrm{A} / \mathrm{B} \\
\mathrm{A} / \mathrm{B} \\
\mathrm{A} / \mathrm{B} \\
\mathrm{A} / \mathrm{B} \\
\mathrm{A} / \mathrm{B} \\
\mathrm{A} / \mathrm{B} \\
\mathrm{A} / \mathrm{B}\end{array}$ & $\begin{array}{l}476 / 400 \\
4599422 \\
4699396 \\
475 / 390 \\
477 / 403 \\
461 / 438 \\
474417 \\
477 / 418 \\
\end{array}$ & $\begin{array}{l}5 / 81 \\
7 / 45 \\
5 / 76 \\
4 / 89 \\
5 / 80 \\
5 / 28 \\
8 / 64 \\
8 / 67\end{array}$ & $\begin{array}{r}89 / 9 \\
85 / 14 \\
74 / 69 \\
119 / 44 \\
63 / 60 \\
136 / 54 \\
75 / 51 \\
70 / 50 \\
\end{array}$ & 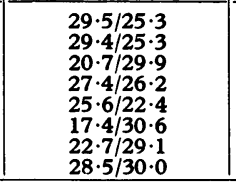 & 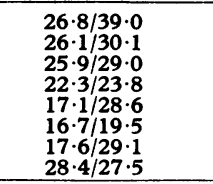 & 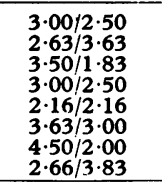 \\
\hline \multicolumn{9}{|c|}{ Subjects $C$ and $D$} \\
\hline 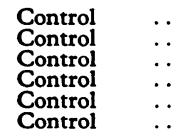 & $\begin{array}{c}: \\
\because \\
\because \\
:\end{array}$ & $\begin{array}{l}\text { C/D } \\
\text { c/D } \\
\text { c/p } \\
\text { c/D } \\
\text { c/D }\end{array}$ & $\begin{array}{l}448 / 440 \\
400 \mid 4118 \\
449144 \\
463 \mid 449 \\
4151451 \\
428 / 407\end{array}$ & $\begin{array}{l}24 / \\
9 / \\
10 / 2 \\
12 / 11 \\
13 / 18 \\
15 / 11\end{array}$ & $\begin{array}{l}53 / 72 \\
60 / 75 \\
46 / 144 \\
66 / 97 \\
74 / 118 \\
62 / 82\end{array}$ & 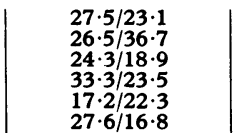 & 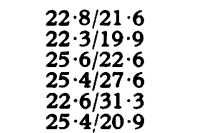 & $\begin{array}{l}2.00 / 1.50 \\
1.831 .50 \\
22.001 .33 \\
11.501 .33 \\
2.331 .33 \\
1.831 .83 \\
1.833\end{array}$ \\
\hline $\begin{array}{lll}\text { Drug } & \text { II } \\
\text { Drug } & \text { II } \\
\text { Drug } & \text { III } \\
\text { Drug } & \text { Iv } \\
\text { Drug } & \text { vil } \\
\text { Drug } & \text { vI } \\
\text { Drug } & \text { IX } \\
\text { Drug } & \text { XI } \\
\text { Drug } & \text { XIII } \\
\text { Drug } & \text { XIV }\end{array}$ & \begin{tabular}{|c|}
.$:$ \\
$\because$. \\
$\because$. \\
$\because$. \\
.
\end{tabular} & $\begin{array}{l}\text { c/D } \\
\text { c/p } \\
\text { c/p } \\
\text { c/p } \\
\text { c/p } \\
\text { c/p } \\
\text { c/p } \\
\text { c/p } \\
\text { c/D }\end{array}$ & 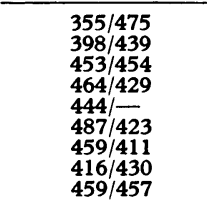 & 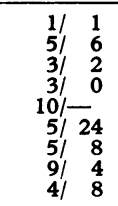 & 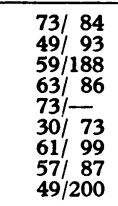 & 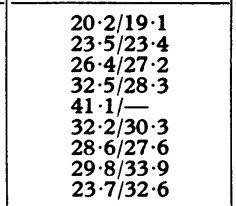 & 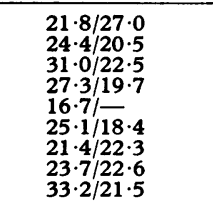 & 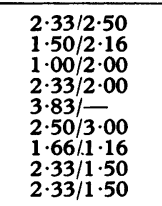 \\
\hline 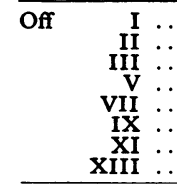 & \begin{tabular}{l|}
.. \\
$\because$. \\
$\because$. \\
.. \\
\end{tabular} & $\begin{array}{l}\text { c/D } \\
\text { c//D } \\
\text { c/D } \\
\text { c/p } \\
\text { c/D } \\
\text { c/p } \\
\text { c/D }\end{array}$ & 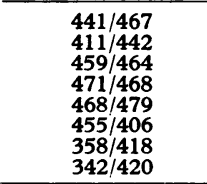 & $\begin{aligned} & 17 / 14 \\
& 9 / 24 \\
& 15 / 10 \\
& 8 / 11 \\
& 15 / 3 \\
& 11 / 15 \\
& 15 / 64 \\
& 20 / 2 \\
& 20 / 2\end{aligned}$ & $\begin{array}{l}50 / 78 \\
69 / 51 \\
52 / 96 \\
49 / 85 \\
55 / 96 \\
50 / 76 \\
75 / 51 \\
62 / 81\end{array}$ & $\begin{array}{l}36.8 / 20 \cdot 8 \\
25 \cdot 1 / 30.2 \\
34.127 .9 \\
30.126 .7 \\
33.824 .4 \\
31.2126 .7 \\
29 \cdot 0.025 .1 \\
31 \cdot 2 / 30 \cdot 3\end{array}$ & 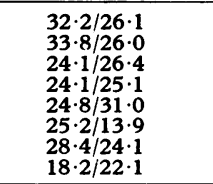 & 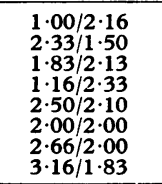 \\
\hline
\end{tabular}

amylobarbitone (Evans et al., 1968). Though $0.8 \mathrm{~g}$. of chloral hydrate did significantly depress R.E.M. sleep in four subjects over the first two to three days, no definite rebound of R.E.M. sleep was observed when the drug was stopped.

Mandrax (Subjects A and B) did produce occasional nights of low R.E.M. sleep time, but the effect was erratic. On only one night for Subjects $C$ and $D$ was R.E.M. time reduced, and the effect of dichloralphenazone was to make the fluctuations in R.E.M. sleep time more apparent. No rebound of R.E.M. sleep was seen when either of these drugs was withdrawn. This result differs from that of Kales et al. (1969), who found that $1 \mathrm{~g}$. of chloral hydrate failed to have any significant effects on R.E.M. sleep, but partly confirms his finding that $150 \mathrm{mg}$. of methaqualone had no significant effects on R.E.M. sleep. Nevertheless, he found that $300 \mathrm{mg}$. of methaqualone did depress R.E.M. sleep significantly. These observations suggest that in order to depress R.E.M. sleep a given drug may need to exceed a "threshold" which may vary in species, individuals, and over a period of time. The threshold for methaqualone may be between 250 and $300 \mathrm{mg}$. In our sub-

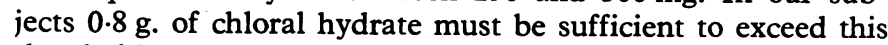
threshold, though Kales et al. found that $1 \mathrm{~g}$. was insufficient.

Further statistical analysis shows evidence of individual differences within the group on chloral. The R.E.M. sleep depression in Subjects $A$ and $B$ is significant at a level of $P<0.01 \quad(t=59.56)$. In the case of Subjects $C$ and $D$ the depression is less significant $(P>0.05, t=13.6222)$. There is no evidence that this is a dose/weight effect.

Thresholds-that is, dose response effects-have been found in administration of other drugs not primarily hypnotics; mono-amine oxidase inhibitors (Akindele et al., 1970), chlorpromazine (Lewis and Evans, 1969), and chlormethiazole (Evans and Lewis, 1968). The existence of such a threshold varying in individuals may explain the conflicting results obtained from various studies of non-barbiturate hypnotics.

The absence of a consistent R.E.M. sleep rebound when these hypnotics were stopped supports the fact that R.E.M. sleep is only minimally affected by clinical doses of these hypnotics. In itself it is not an absolute indication that no clinical withdrawal complaints arose. In this study, however, it was found that no definite disturbance of slow-wave sleep occurred when the drugs were stopped.

An excess of R.E.M. sleep and a disturbance of slow-wave sleep can be tolerated by subjects without definite complaint (Evans et al., 1968), but if the increase in R.E.M. sleep is severe and intense, nightmares occur, as Oswald and Priest (1965) showed when sodium amylobarbitone $(600 \mathrm{mg}$.) was withdrawn. Kales and Jacobson (1967) recorded nightmares when methyprylone (300 mg.) was stopped. Comparison of the studies of sodium amylobarbitone $200 \mathrm{mg}$. (Evans et al., 1968) and $600 \mathrm{mg}$. (Oswald and Priest, 1965) shows that the degree and duration of the recorded sleep disturbance were dependent on the dose.

While it may be that withdrawal of a dose of these nonbarbiturate hypnotics sufficient to seriously exceed the suggested threshold will result in measurable and clinical signs and symptoms, this is not yet proved. 


\section{REFERENCES}

Akindele, M. O., Evans, J. I., and Oswald, I. (1970). In press. de Clérambault, G. (1910). Annales Médico-Psychologiques, 11, 33. Davison, K. (1969). British Medical Fournal, 1, 781

Dement, W., and Kleitman, N. (1957). Electroencephalography and Clinical Neurophysiology, 9,673 .

Evans, J. I., and Lewis, S. A. (1968). Unpublished data.

Evans, J. I., and Lewis, S. A. (1968), Unpublished data.
Evans, J. I., Lewis, S. A., Gibb, I. A. M., and Cheetham, M. (1968). British Medical fournal, 4, 291.

Greenberg, R., and Pearlman, C. (1967). American fournal of Psychiatry, 124, 133.

Gresham, S. C., Webb, W. B., and Williams, R. L. (1963). Science, 140, 1226 .

Gross, M. M., et al. (1966). Journal of Nervous and Mental Disease, 142, 493.

Johnson, J., and Clift, A. D. (1968). British Medical fournal, 4, 613.

Jouvet, M.'(1967). Physiological Reviews, 47, 117.
Kales, A., and Jacobson, A. (1967). Experimental Neurology, Suppl., 4, 81. Kales, A., Jacobson, A., Kales, J. D., Marusak, C., and Hanley, J. (1968). Psychophysiology, 4, 391.

Kales, A., et al. (1969). Annals of Internal Medicine, 70, 591.

Lehmann, H. E., and Ban, T. A. (1968). International fournal of Clinical Pharmacology, Therapy and Toxicology, $1,424$.

Lewis, S. A., and Evans, J. I. (1969). Psychopharmacologia, 14, 342

Mendels, J., and Hawkins, D. R. (1967). Electroencephalography and Clinical Neurophysiology, 22, 556 .

Oswald, I., Evans, J. I., and Lewis, S. A. (1969). In Scientific Basis of Drug Dependence, ed. H. Steinberg. London, Churchill.

Oswald, I., and Priest, R. G. (1965). British Medical fournal, 2, 1093.

Rechtschaften, A., and Kales, A. (editors) (1968). Manual of Standardized Terminology, Techniques and Scoring System for Sleep Stages of Human Subjects. Washington, D.C., U.S. Government Printing Office.

Tissot, R. (1965). Progress in Brain Research, 18, 175.

\title{
Osteotomy of the Lesser Trochanter for Rheumatoid Arthritis of the Hip
}

\author{
J. A. FIXSEN,* M.CHIR., F.R.c.s. ; M. F. SULLIVAN, † F.R.c.s.
}

British Medical fournal, 1970, 3, 313-315

Summary: Forty-eight operations of osteotomy of the lesser trochanter in 45 patients were reviewed after an average of three and a half years. Of 10 hips affected by proved rheumatoid arthritis eight obtained complete relief of pain and two partial relief. Of 38 hips affected by osteoarthritis 16 had complete relief and 17 partial relief.

The results suggest that osteotomy of the lesser trochanter deserves a more extended trial in cases of proved rheumatoid arthritis of the hip joint.

\section{Introduction}

Release of the pelvifemoral musculature for relief of pain in osteoarthritis of the hip was introduced by Voss (1956). In his original operation he performed a fasciotomy of the iliotibial tract, a basal osteotomy of the greater trochanter, and a wide subcutaneous adductor tenotomy. This was quite an extensive procedure and O'Malley (1959) suggested that release of the iliopsoas muscle was a more important factor. Layani, Cordier, Garnier, Roeser, and Paquet (1959) injected 1\% lignocaine into various sites of proposed muscle division and found that the greatest relief of pain occurred from injection around the insertion of the iliopsoas. They followed this with a clinical trial of osteotomy of the lesser trochanter added to the Voss procedure (Cordier, Layani, and Garnier, 1960). Their series included four cases of protrusio acetabulae with complete relief of pain.

Following this work a prospective clinical trial was started by Mr. J. N. Wilson in 1961. Osteotomy of the lesser trochanter was performed for cases of painful arthritis of the hip, both osteoarthritis and rheumatoid arthritis. We have not found any previous report of an operation of this nature for rheumatoid arthritis, which is the main subject of this communication.

\section{Operation and Aftercare}

The patient, under general anaesthesia, is placed in the lateral position with the affected hip uppermost. A vertical incision $10 \mathrm{~cm}$. long is made just medial to the posterior surface of the great trochanter and upper part of the shaft of the femur. This incision resembles the lower part of Austin Moore's posterior approach to the hip joint. The fascia lata is divided in the line of the incision. The lower limb is rotated inwards as far as possible and the quadratus femoris is divided near its insertion with diathermy. This muscle is retracted medially, exposing the lesser trochanter, which is * Orthopaedic Registrar.

+ Registrar.
Institute of Orthopaedics and the Royal National Orthopaedic Hospital, London. divided with a broad osteotome. The osteotome should be directed anteromedially so as to cut the lesser trochanter flush with the femur. The trochanter is immediately raised 2 to 3 $\mathrm{cm}$. by tension of the iliopsoas. The wound is closed with Redon suction drainage. If the adductors are tight subcutaneous tenotomy is performed.

After the operation the patient lies free in bed until the sutures are removed and is then allowed up and about. The postoperative radiograph shows the lesser trochanter freed and at a higher level. Very little is required in the way of physical treatment, and the average time spent in hospital is two and a half weeks.

\section{Clinical Material}

In this series osteotomy of the lesser trochanter was carried out on 50 patients. Forty-five-19 men and 26 womenattended for follow-up and review of their 48 operations. Their average age at the time of operation was 61 years, range 36 to 78 . The time of follow-up was one to seven years, mean three and a half years.

Of the 48 hips 30 were thought to have primary or idiopathic osteoarthritis, 10 rheumatoid arthritis, and eight secondary osteoarthritis. To be classified as rheumatoid arthritis cases had to show radiographic changes in the hands as well as the hip, a raised erythrocyte sedimentation rate, and a positive latex fixation test.

\section{Results}

The results have been analysed in three groups, according to the aetiology. The results were assessed by considering relief of pain, functional activity, movement of the hip, and radiographic appearances.

Relief of pain.-Pain was the main reason for operation. The relief of pain has been classified into complete relief, partial relief, and no relief (Table I). Patients with complete relief were free from pain from the time of operation to follow-up. Those with partial relief were initially pain-free and then had recurrence; the average time of recurrence was one year after operation. In the two cases of rheumatoid arthritis pain recurred at 12 and 14 months; this pain was less than before operation and neither patient felt that further treatment was necessary.

TABle I

\begin{tabular}{|c|c|c|c|c|c|c|}
\hline & & & & $\begin{array}{c}\text { Complete } \\
\text { Relief }\end{array}$ & $\begin{array}{l}\text { Partial } \\
\text { Relief }\end{array}$ & $\begin{array}{l}\text { No } \\
\text { Relief }\end{array}$ \\
\hline $\begin{array}{l}\text { Primary osteoarthritis .. } \\
\text { Rheumatoid arthritis . } \\
\text { Secondary osteoarthritis.. }\end{array}$ & $\begin{array}{l}\cdots \\
\because \\
\end{array}$ & $\begin{array}{l}\ldots \\
\cdots\end{array}$ & $\begin{array}{l}. . \\
\therefore\end{array}$ & $\begin{array}{r}13 \\
8 \\
3\end{array}$ & $\begin{array}{r}14 \\
2 \\
3\end{array}$ & $\begin{array}{l}3 \\
\mathbf{0} \\
2\end{array}$ \\
\hline
\end{tabular}

